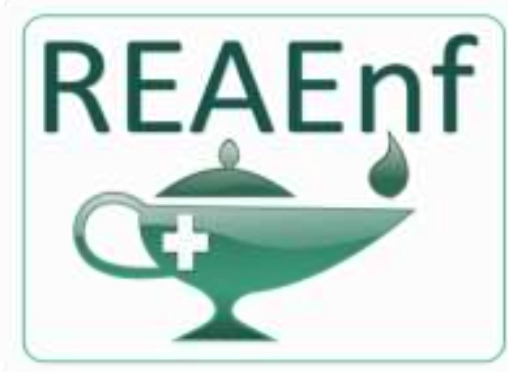

Revista Eletrônica Acervo Enfermagem

\section{ARTIGO ORIGINAL}

Recebido em: 8/2019

Aceito em: 8/2019

Publicado em: 9/2019

\title{
A atuação da enfermagem na oficina terapêutica no contexto de um centro de atenção psicossocial
}

\author{
Nursing performance in the therapeutic workshop in the context of a psychosocial care \\ center
}

Desempeño de enfermería en el taller terapéutico en el contexto de un centro de atención psicosocial

Iel Marciano de Moraes Filho ${ }^{1 *}$, Luiza Monteiro de Moura², Alessandra Mateus Souza ${ }^{2}$, Leidivan Santos Pereira ${ }^{2}$, Luciana Clemente Nicácio Santos ${ }^{2}$, Aline Aparecida Arantes ${ }^{3}$, Francidalma Soares Sousa Carvalho Filha ${ }^{2}$, Gabriel Ilode Alsteen², Talita Vanderlei Da Silva De Sousa ${ }^{4}$, Mayara Cândida Pereira1.

\begin{abstract}
Resumo: Esse artigo buscou relatar o funcionamento de uma oficina de arteterapia institucionalizada por uma enfermeira, e logo como a mesma, inserida dentro de um Centro de Atenção Psicossocial II, pode ser importante no tratamento das psicopatologias mentais, através da realização de trabalhos artesanais. $O$ estudo foi conduzido em um Centro de Atenção Psicossocial II, de um município no entorno do Distrito Federal - DF. Adotou-se um método misto de pesquisa, mediante a combinação de abordagens quantitativa e qualitativa na investigação, caracterizando-se pelo desenho sequencial explanatório. A oficina resgata e incentiva os usuários a olharem para o mundo através de suas produções artísticas e proporciona troca de vivencias com os demais usuários do CAPS, demonstrando as suas emoções e seus sentimentos, antes contidos, e no momento, verbalizados para o grupo, abrangendo a criatividade nas pinturas, nas escolhas de cores e nas trocas de experiências. Sendo assim, os profissionais atuantes nos CAPS necessitam ampliar as suas interfaces de conhecimento para abarcar estes novos métodos que são de grande valia no cuidado a pessoa com transtorno mental, diminuindo a medicalização e proporcionando estratégias de enfrentamento.
\end{abstract}

Palavras-chave: Intervenção Psicossocial; Enfermagem Psiquiátrica; Saúde Mental.

\begin{abstract}
This article aimed to report the operation of an art therapy workshop institutionalized by a nurse, and soon as the same, inserted within a Psychosocial Care Center II, can be important in the treatment of mental psychopathologies, through the execution of artisanal works. The study was conducted in a Psychosocial Care Center II, in a city around the Federal District - DF. A mixed research method was adopted, by combining quantitative and qualitative research approaches, characterized by the sequential explanatory
\end{abstract}

\footnotetext{
${ }^{1}$ Universidade Paulista (UNIP), Brasília - DF. *E-mail: ielfilho@yahoo.com.br

${ }^{2}$ Faculdade de Ciências e Educação Sena Aires (FACESA), Valparaiso de Goiás - GO.

${ }^{3}$ Faculdade União de Goyazes (FUG), Trindade- GO.

4 Universidade Estadual do Maranhão (UEMA), Balsas - MA.
} 
design. The workshop rescues and encourages users to look at the world through their artistic productions and provides an exchange of experiences with other CAPS users, demonstrating their previously contained and verbalized emotions to the group, including creativity in paintings, in color choices and in the exchange of experiences. Thus, professionals working in CAPS need to broaden their knowledge interfaces to embrace these new methods that are of great value in the care of people with mental disorders, reducing medicalization and providing coping strategies.

Keywords: Psychosocial Intervention; Psychiatric nursing; Mental health.

Resumen: Este artículo tuvo como objetivo informar la operación de un taller de terapia de arte institucionalizado por una enfermera, y tan pronto como el mismo, insertado dentro de un Centro de Atención Psicosocial II, puede ser importante en el tratamiento de las psicopatologías mentales, a través de la ejecución de trabajos artesanales. El estudio se realizó en un Centro de Atención Psicosocial II, en una ciudad alrededor del Distrito Federal - DF. Se adoptó un método de investigación mixto, combinando enfoques de investigación cuantitativa y cualitativa, caracterizados por el diseño explicativo secuencial. El taller rescata y alienta a los usuarios a mirar el mundo a través de sus producciones artísticas y proporciona un intercambio de experiencias con otros usuarios de CAPS, demostrando al grupo sus emociones previamente contenidas y verbalizadas, incluyendo creatividad en pinturas, en elecciones de colores y en el intercambio de experiencias. Por lo tanto, los profesionales que trabajan en CAPS necesitan ampliar sus interfaces de conocimiento para adoptar estos nuevos métodos que son de gran valor en el cuidado de personas con trastornos mentales, reduciendo la medicalización y proporcionando estrategias de afrontamiento.

Palabras clave: Intervención psicosocial; Enfermería psiquiátrica; Salud mental.

\section{INTRODUÇÃO}

Os Centros de Atenção Psicossocial (CAPS), foram criados como serviços regionalizados integrando a rede de atenção à Saúde Mental, proporcionam atendimento à população em todo o território nacional. Perfazem a uma nova modalidade de atenção implantada no Sistema Único de Saúde (SUS) que envolve a construção de uma política pública baseada em práticas multiprofissionais pautadas na interdisciplinaridade (BRASIL, 2004; NASCIMENTO AF e GALVANESE ATC, 2009). São considerados dispositivos estratégicos na desinstitucionalização das pessoas com transtornos mentais, sendo assim, serviços substitutivos ao modelo asilar de cuidado em saúde mental, e estão fundamentados na noção de apoio matricial além de possuírem políticas públicas comuns como a Atenção Básica (NUNES M, et. al, 2009).

Os mesmos atendem os pacientes através de suas reais necessidades que são potencializadas através dos Projetos Terapêuticos Singulares (PTS). Portanto os próprios se configura, como uma ferramenta que possibilita um cuidado integral e singular, na atuação de modo holístico, que valoriza todas as características da pessoa em tratamento, e não foca apenas no diagnóstico psiquiátrico, e nos medicamentos prescritos para o tratamento dos usuários, vai muito além pautando em contribuições técnicas dos integrantes da esquipe interdisciplinar, juntamente com os familiares, usuários e articulações intersetoriais que favorecem estas ações (BRASIL, 2004; MORAES FILHO IM, et al., 2015).

Com a aprovação da lei no 9.867, de 10 de novembro de 1999, houve um incentivo para que as oficinas terapêuticas começassem a ser criadas dentro dos CAPS (FARIAS ID, et al., 2016; BRASIL, 2004). As mesmas constituem-se em importantes espaços para a reabilitação de pessoas com transtornos mentais, nos quais as ações operacionais dos CAPS podem ser concretizadas (ALMEIDA N, 2004). Atualmente são compostas por atividades diversas, inclusive arte e artesanato (LAPPANN-BOTTI NC e LABATE RC, 2004).

Desta forma uma das práticas difundidas dentro dos PTS e a arteterapia, a mesma se configura como uma especialidade destinada a profissionais com graduação na área da saúde, como Psicologia, Enfermagem e Fisioterapia, embora se reconheça sua utilização por pessoas formadas nas áreas das artes e da educação, desde que sem o enfoque clínico (REIS AL, 2014). Também, tem sido considerada como uma modalidade 
terapêutica com características próprias, diferenciando-se através de duas linhas de atuação: arte como terapia, onde o foco principal está no processo artístico levando em consideração suas propriedades curativas, e arte psicoterapia, utilizando a arte para ajustes psicoterapêuticos específicos, seguindo princípios, técnicas e teorias visando o desenvolvimento emocional do indivíduo (ANDRADE LQ, 2001). O seu crescimento interior, abre novos horizontes, proporcionando mudanças psíquicas, expandindo a consciência, promovendo a reconciliação de conflitos emocionais, o autoconhecimento e o desenvolvimento pessoal (VASCONCELLOS EA e GIGLIO JS, 2007).

O presente estudo teve como objetivo relatar o funcionamento de uma oficina de arteterapia institucionalizada por uma enfermeira, e logo como a mesma, inserida dentro de um CAPS II, pode ser importante no tratamento das psicopatologias mentais, através da realização de trabalhos artesanais. $O$ presente estudo se justifica devido à importância inerente das oficinais de arteterapia, no tratamento de pessoas com transtorno mentais e no favorecimento das práticas integrativas e completares na diminuição da medicalização.

\section{MÉTODOS}

Este estudo foi conduzido em um CAPS II, de um município no entorno do Distrito Federal - DF. Adotouse um método misto de pesquisa, mediante a combinação de abordagens quantitativa e qualitativa na investigação, caracterizando-se pelo desenho sequencial explanatório (TASHAKKORI A e TEDDLIE C 1998; O'CATHAIN A, et al., 2008; CRESWELL JW, PLANOCLARK VL, 2007).

Na primeira etapa quantitativa, a população do estudo incluiu todos os pacientes que faziam atendimento no CAPS II e participavam da oficina de arteterapia, com a identificação das patologias pautadas na Classificação Internacional de Doenças (CID) versão 11, da Organização Mundial de Saúde (OMS) de maior incidência (OMS, 2019). O tamanho da amostra foi de 1881 atendimentos no período de janeiro de 2018 a janeiro de 2019. Os dados foram coletados em base de dados secundários, cedidos pela a Secretaria Municipal de Saúde através do método de pesquisa documental, quantificando os atendimentos.

$\mathrm{Na}$ etapa qualitativa subsequente, a população do estudo incluiu a oficina de arteterapia comandado por uma enfermeira onde o estudo fora sedimentado em relato de experiência, o mesmo fora realizado por alunos da pós-graduação lato Sensu em Saúde Mental. Através da observação não participante dos usuários, fora elencado as ações dos mesmos e as percepções dos pesquisadores referentes as práticas desenvolvidas.

O presente Trabalho respeitou todas as determinações da resolução 466/2012 do Conselho Nacional de Saúde, em suas duas partes, o mesmo dispensa-se do parecer de um Comitê de Ética e pesquisa, por se tratar apenas de dados de domínio público e a percepção dos pesquisadores do estudo de forma que não houve a identificação dos participantes.

Os dados foram analisados por teste de média e quando significativos foram utilizados, o teste de Tukey a $5 \%$ em amostra independente e múltiplas variáveis através da análise de variância (ANOVA). Os dados foram organizados em planilhas do Microsoft Excel® e analisados pelo programa estatístico Sisvar ${ }^{\circledR}$ versão 5.6. para Windows $\AA^{\circledR}$.

\section{RESULTADOS E DUSCUSSÃO}

Os CAPS II fornecem atendimento a todas as faixas etárias, para transtornos mentais graves e persistentes, inclusive pelo uso de substâncias psicoativas, atendendo cidades ou regiões com pelo menos 70 mil habitantes (MARTINHAGO F e OLIVEIRA WF, 2012; MORAES IM, et al., 2015).

Segundo a análise de variância em relação a ocorrência das principais patologias atendidas no CAPS II de um município no entorno de Brasília DF, tornou-se significativa estatisticamente (Tabela 1). Em relação ao decorrer do ano, o maior índice de atendimentos foi relacionado à exames de rotina (Z000), transtorno afetivo bipolar, episódio atual maníaco com sintomas psicóticos ( $F 32$ )), transtorno misto ansioso e depressivo ( $F$ 412 ) e esquizofrenia paranoide ( $F 20$ )), totalizando 1881 atendimentos em geral no ano. 
Tabela 1 - Principais patologias atendidas no CAPS II de um municio no entorno do Distrito Federal no período de janeiro/18 a janeiro/19.

\begin{tabular}{|c|c|c|c|c|c|c|c|c|c|c|c|c|c|}
\hline Tratamentos $^{\circ}$ & Jan/18 & Fev/18 & Mar/18 & Abr/18 & Mai/18 & Jun/18 & Jul/18 & Ago/18 & Set/18 & Out/18 & Nov/18 & Dez/18 & Jan/19 \\
\hline Z000 & $94 \mathrm{a}$ & $53 \mathrm{a}$ & $68 a$ & $55 a b$ & $24 a b$ & $46 b$ & $64 a$ & $83 a$ & $15 b c$ & $3 b c$ & $4 \mathrm{bc}$ & $3 c$ & Od \\
\hline F 200 & $22 \mathrm{bc}$ & $0 d$ & $30 b$ & $26 \mathrm{~b}$ & $1 \mathrm{c}$ & $14 b c$ & $12 b c$ & $15 b c$ & $10 \mathrm{bc}$ & $9 b$ & $18 \mathrm{bc}$ & $17 \mathrm{bc}$ & $20 \mathrm{bc}$ \\
\hline F 320 & $37 b$ & $40 \mathrm{~b}$ & $37 b$ & $56 \mathrm{a}$ & $46 \mathrm{a}$ & $64 a$ & $41 a b$ & $32 b$ & $22 \mathrm{bc}$ & $23 a$ & $32 a b$ & $16 \mathrm{bc}$ & $10 \mathrm{bc}$ \\
\hline F 322 & $23 b c$ & $11 b c$ & $7 b c$ & $24 \mathrm{bc}$ & $17 a b$ & $28 b c$ & $14 b c$ & $6 b c$ & $9 b c$ & $13 b c$ & $14 b c$ & $15 b c$ & $41 \mathrm{~b}$ \\
\hline F 412 & $20 b c$ & $14 b c$ & $14 \mathrm{bc}$ & $50 a b$ & $27 a b$ & $50 b$ & $20 b$ & $24 b c$ & $43 \mathrm{a}$ & $0 d$ & $51 \mathrm{a}$ & $36 a$ & $57 \mathrm{a}$ \\
\hline F 600 & $2 \mathrm{c}$ & $3 c$ & $2 d$ & $2 b c$ & $5 c$ & $3 c$ & $2 c$ & $2 c$ & $3 c$ & $2 b c$ & $2 \mathrm{c}$ & $2 \mathrm{c}$ & $7 \mathrm{bc}$ \\
\hline F 700 & $3 b c$ & $3 c$ & $3 c$ & $7 b c$ & $3 c$ & $4 b c$ & $2 c$ & $2 c$ & $2 c$ & $2 b c$ & $3 c$ & $1 d$ & $5 c$ \\
\hline F 790 & $0 d$ & $4 b c$ & $2 d$ & $1 d$ & $0 \mathrm{~d}$ & $1 d$ & $1 d$ & $1 d$ & $1 d$ & $1 c$ & $0 \mathrm{~d}$ & $1 d$ & $1 c$ \\
\hline CV (\%) & 0.0 & 0.0 & 0.0 & 0.0 & 0.0 & 0.0 & 0.0 & 0.0 & 0.0 & 0.0 & 0.0 & 0.0 & 0.0 \\
\hline FV & \multicolumn{13}{|c|}{ (Anova Probabilidade do teste de F) } \\
\hline Tratamento & $0.0^{*}$ & $0.0^{*}$ & $0.0^{*}$ & $0.0^{*}$ & $0.0^{*}$ & $0.0^{*}$ & $0.0^{*}$ & $0.0^{*}$ & $0.0^{*}$ & $0.0^{*}$ & $0.0^{*}$ & $0.0^{*}$ & $0.0^{*}$ \\
\hline
\end{tabular}

Fonte: Dados da pesquisa, 2019.

*médias significativas ao Teste de Tukey para $p<0,01$.

* a, b, c nível gradual de significância do teste estatístico.

-Tratamentos: (Z000) exame de rotina; ( F 20 0) esquizofrenia paranoide; ( $F 32$ 0) transtorno afetivo bipolar, episódio atual maníaco com sintomas psicóticos; ( F 32 2) episódio depressivo grave sem sintomas psicóticos; ( F 412 2) transtorno misto ansioso e depressivo; ( $F$ 60 0) personalidade paranoica; (F70 0) retardo mental leve; (F79 0) retardo mental não especificado- menção de ausência ou de comprometimento mínimo do comportamento. 
A aplicação da arteterapia com diferentes patologias de caráter psicossociais, proporcionam aos profissionais um momento de reflexão para reconhecer o estado emocional dos seus participantes e ajuda de maneira satisfatória na remissão de sintomas depressivos, o que facilita no processo de reabilitação dos usuários (VALLADARES-TORRES ACA, 2017).

As oficinas terapêuticas podem oportunizar um espaço de criação através da realização de atividades manuais ou mecânicas e oportunizam um espaço de convivência para pessoas com transtornos mentais (GALLETTI MC, 2004).

Ao analisarmos o número total de consultas no período de janeiro de 2018 a janeiro de 2019, no CAPS II de uma cidade no entorno do DF, o gráfico demonstra maior acesso no CAPS II, totalizando 1881 atendimentos. Enquanto na Oficina de arteterapia coordenada pela enfermeira, totalizou 815 atendimentos durante esse período (Gráfico 1). Ao comparamos as consultas no decorrer do ano, no CAPS II o mês de maior relevância foi abril de 2018, chegando a 221 consultas. E o menor índice estabeleceu-se ao mês de outubro de 2018.

Gráfico 1 - Comparação referente ao total de consultas das principais patologias, no período de janeiro/2018 a janeiro/2019, aos atendimentos na Oficina de arteterapia da enfermeira no CAPS II localizado em uma cidade no entrono do DF, 2019.

\section{Total de consultas das principais patologias no período de Jan/18 a Jan/19}

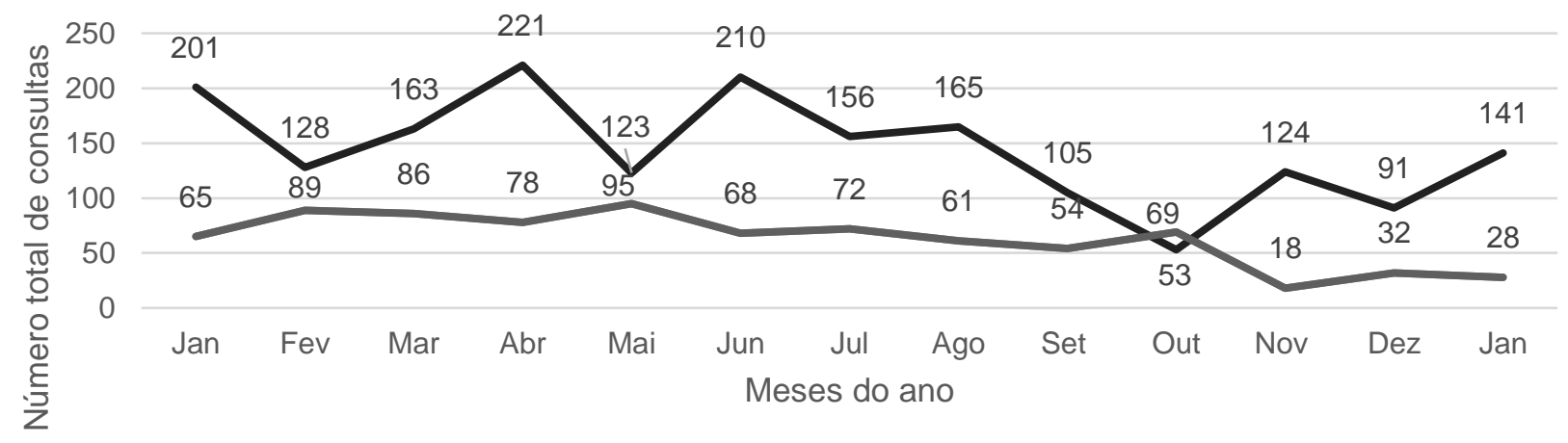

CAPS Oficina de arte terapia

Fonte: Dados da pesquisa, 2019.

Aos valores referentes a Oficina de arteterapia, o mês de maior índice de atendimento, se deu ao mês de maio de 2018, totalizando 95 atendimentos. Enquanto o de menor frequência refere-se a novembro de 2018, totalizando 18 atendimentos. O gráfico demonstra que o acesso ao CAPS II é maior em relação a Oficina de arteterapia, com exceção do mês de outubro, em que a oficina obteve maior índices de atendimentos em relação ao CAPS II.

As oficinas contribuem para que os pacientes realizem algumas reflexões, expondo seus desejos, fortalecendo seus laços e atitudes que podem colaborar para a melhora das relações familiares, objetivando estimular a autonomia, dos integrantes do grupo, como propõe a Psicologia Social Comunitária (HAYGERT SCM e PASETTO MI, 2015).

A oficina de arterapia atendeu $43,32 \%$ do público total do CAPS II durante o período de um ano, as oficinas e grupos terapêuticos são considerados como um das principais formas de tratamento dos CAPS, com o objetivo de promover a integração social e familiar, a expressão dos sentimentos e dificuldades, o desenvolvimento de habilidades pessoais e laborais, e o exercício da cidadania promovem qualidade de vida aos usuários (BRASIL, 2004a). Assim as práticas de grupos e oficinas fazem parte do cotidiano dos profissionais e usuários dos CAPS (MARTINHAGO F e OLIVEIRA WF, 2012). 
Na oficina de arteterapia são desenvolvidas pela enfermeira e os usuários técnicas de crochê, tricô, fuxico, patchwork, pintura em telas e em panos de prato, biscuit, trabalhos manuais com materiais reciclados, costura e dobraduras em papeis, as atividades proporcionavam aos usuários melhora significativa através da oficina e também e um momento oportuno para orientações de caráter inerentes a educação em saúde através de: orientações a infecções sexualmente transmissíveis, exames de rastreamento, medicações e logo os seus efeitos adversos.

Após a oficina os mesmos conseguiram se desinibir perante ao grupo melhorando a interação social, diminuição da psicomotricidade, melhora da concentração, coordenação motora, compartilhamento de vivências e experiências, raciocínio lógico, domínio de habilidades de autocontrole e autodomínio, desta forma grande parte dos usuários atendidos conseguiram através da mesma diminuírem os efeitos ocasionados pelos psicotrópicos e as limitações advindas das psicopatologia, os mesmos se mostraram mais felizes, através da melhora da qualidade das relações interpessoais tanto no âmbito social quanto familiar .

A oficina resgata e incentiva os usuários a olharem para o mundo através de suas produções artísticas e proporciona troca de vivencias com os demais usuários do CAPS, demonstrando as suas emoções e seus sentimentos, antes contidos, e, no momento, verbalizados para o grupo, abrangendo a criatividade nas pinturas, nas escolhas de cores e nas trocas de experiências. O objetivo da oficina de arterapia é proporcionar uma reconstrução do cotidiano dos pacientes a partir de ações terapêuticas, que podem levar não somente à ressignificação da vida, mas também ha atribuição de novos sentidos para longevidade da mesma (SANTOS MM, et al., 2016).

Assim, de forma geral, pode-se afirmar que o papel fundamental da Enfermagem nos CAPS está voltado ao direcionamento da assistência integral, dependendo das necessidades e da capacidade criativa de cada equipe dos serviços. Para o desnovelar desta prática e necessário implementar um modo de lidar com o usuário que não é apenas a "soma" do conhecimento das diferentes profissões que compõem o quadro de funcionários do CAPS, mas a produção de uma forma de cuidar do usuário a partir da contribuição de todos, e, portanto, interdisciplinar na prática, e sob o enfoque do autocuidado de maneira holística. A profissão para atuar neste serviço, deverá estar sempre aberta para novas possibilidades, como as desenvolvidas pela a enfermeira na oficina de arteterapia (MORAES IM et al., 2015; MORAES IM et al., 2015; Moraes-Filho IM, et al.,2019).

As oficinas terapêuticas proporcionam um momento raro no cotidiano dos serviços e dos envolvidos com o mesmo, além de expandir os lugares dos usuários e do próprio serviço. As mesmas necessitam de critérios para a avaliação no requisito de extensão de direitos e cidadania, assim como inclusão social dos sujeitos com sofrimento psíquico. Os projetos culturais na interface com a saúde e cultura têm como objetivo desenvolver e classificar os processos de promoção da saúde por meio de atividades culturais, reconhecendo o ser humano como sujeito integral e a saúde como qualidade de vida (MELO NS, 2009).

As mesmas foram institucionalizadas por Osório César, que era um profissional médico sanitarista, a frente do seu tempo e com ideais pautados no modelo biopsicossocial, o mesmo é o precursor de referência no que diz respeito à terapia realizada através de atividades por meio de oficinas (FARIAS ID, et al.,2016). Em 1946, a psiquiatra Nise da Silveira propôs o uso de atividades diversas, dentre elas, as artísticas, como recurso terapêutico, assim se aproximando do que chamamos hoje de oficina terapêutica (MELO W, 2001).

\section{CONCLUSÃO}

As oficinas de arteterapia configuram como um instrumento global que ajuda os profissionais atuantes em serviços abertos substitutivos a proporcionarem aos pacientes momentos de reflexão, convivência, exposição dos sentimentos vivenciados, troca de experiências de vida, confronto de realidades oportunizando aos usuários resignação, diminuição de sintomas depressivos e novas perspectivas de vida. Desta forma os profissionais atuantes nos CAPS necessitam ampliar as suas interfaces de conhecimento para abarcar estes novos métodos, que são de grande valia no cuidado a pessoa com transtorno mental, diminuindo a medicalização e proporcionando estratégias de enfrentamento. 


\section{REFERÊNCIAS}

1. ALMEIDA N. Contribuições à tematização das oficinas nos Centros de Atenção Psicossocial. In COSTA CM, FIGUEIREDO AC. (org). Oficinas terapêuticas em saúde mental: sujeito, produção e cidadania. Rio de Janeiro: Contracapa Livraria; 2004.p.167-172.

2. ANDRADE LQ. Terapias expressivas: arte terapia, arte educação, terapia artística. São Paulo: Vetor; 2000.

3. BRASIL. Ministério da Saúde. Secretaria Executiva. Legislação em Saúde Mental 1990-2004. 5a ed. Brasília: Ministério da Saúde; 2004a.

4. BRASIL. Ministério da Saúde. Manual de Saúde Mental. Os Centros de Atenção Psicossocial. Brasília: Ministério da Saúde; 2004b.

5. BRASIL. Ministério da Saúde. Secretaria de Atenção à Saúde: Núcleo Técnico da Política. Nacional de Humanização. Clínica ampliada equipe de referência e projeto terapêutico singular. Brasília: Ministério da Saúde; 2007.

6. CRESWELL JW, PLANOCLARK VL. Designing and conducting mixed methods research. Thousand Oaks: Sage; 2007.

7. FARIAS ID, et.al. A oficina terapêutica como espaço relacional na atenção psicossocial. Rev Uruguaya de Enfermería. Montevideo. 2016; 11(2): 01-13.

8. GALLETTI MC. Oficina em saúde mental: instrumento terapêutico ou intercessor clínico? 1ed.Goiania: editora UCG;2004.

9. HAYGERT SCM, PASETTO ML. Experiência de uma oficina arteterapêutica: uma abordagem multiprofissional no cuidado a saúde mental de idosos;2015; Santa Maria. Anais. Santa Maria: VI Semana Acadêmica Integrada Faculdade Integrada de Santa Maria - FISMA-; 2015.p.149-155.

10. LAPPANN-BOTTI NC, LABATE RC. Oficinas em saúde mental: a representação dos usuários dos serviços de saúde mental. Texto Contexto Enfem. 2004;13(4):519-26.

11. MARTINHAGO F, OLIVEIRA WF. A prática profissional nos Centros de Atenção Psicossocial II (CAPS II), na perspectiva dos profissionais de saúde mental de Santa Catarina. Saúde em Debate [Internet]. 2012;36(95):583594.

12. MELO W. Nise da Silveira e o campo da Saúde Mental (1944-1952): contribuições, embates e transformações. Mnemosine. 2009; 5(2):30-52.

13. MORAES IM, et al. Concepções de enfermeiros de um centro de atenção psicossocial sobre o cuidar a adultos com transtornos mentais graves- relato de experiência. REVISA. 2015; 4(2): 86-95.

14. MORAES-FILHO IM, et al. Atuação dos enfermeiros nos centros de atenção psicossocial- Revisão de Literatura. REVISA. 2015; 4(2):79-93.

15. MORAES-FILHO IM, et al. Retrocesso nas políticas nacionais de saúde mental e de álcool e outras drogas no brasil a partir da nota técnica $n^{\circ} 11 / 2019$. REVISA. 2019; 8(2):115-8.

16. NASC MELO, W. Nise da Silveira. Rio de Janeiro, Imago, 2001.

17. IMENTO AF, GALVANESE ATC. Avaliação da estrutura dos centros de atenção psicossocial do município de São Paulo, SP. Revista de Saúde Pública [Internet]. 2009;43(1):8-15.

18. NUNES M, et al. Ações de saúde mental no Programa Saúde da Família: confluências e dissonâncias das práticas com os princípios das reformas psiquiátrica e sanitária. Cad. Saúde Pública [Internet]. 2007 Oct [cited 2019 June 03]; 23(10): 2375-2384.

19. O'CATHAIN A, et.al. The quality of mixed methods studies in health services research. J Health Serv Res Policy. 2008;13(2):92-8.

20. Organização Mundial da Saúde (OMS). CID-11 3/4 Classificação Internacional de Doenças, décima primeira versão. Genebra: Organização Mundial da Saúde; 2019

21. REIS AC. Arteterapia: a arte como instrumento no trabalho do Psicólogo. Psicologia: Ciência e Profissão, 34(1), 142157. 2014.

22. SANTOS MM, et al. Projetos culturais nos centros de atenção psicossocial: um desafio em direção à cidadania. Cadernos brasileiros de saúde mental.2016; 8(20):114-136

23. TASHAKKORI A, TEDDLIE C. Mixed methodology: combining qualitative and quantitative approaches. Thousand Oaks: Sage Publications; 1998.

24. VALLADARES-TORRES ACA. A contribuição da Arteterapia na remissão de sintomas depressivos e ansiosos nas toxicomanias. Rev. Científica Arteterapia Cores da Vida.2017;24(2):36-35.

25. VASCONCELLOS EA, GIGLIO JS. Introdução da arte na psicoterapia: enfoque clínico e hospitalar. Estudos de Psicologia. 2007 [cited 2019 Apr 26]; 24(3): 375-83. 